\title{
Radiographic outcomes of endoscopic decompression for lumbar spinal stenosis
}

\author{
*Siri Sahib Khalsa, MD, ${ }^{1}$ Hyeun Sung Kim, MD, PhD, ${ }^{2}$ Ravindra Singh, MD, ${ }^{2}$ and \\ Osama Nezar Kashlan, MD1
}

\begin{abstract}
'Department of Neurosurgery, University of Michigan, Ann Arbor, Michigan; and 2Department of Neurosurgery, Nanoori Gangnam Hospital, Seoul, Republic of Korea
\end{abstract}

\begin{abstract}
OBJECTIVE Lumbar central stenosis can theoretically be decompressed with minimal bone removal via an endoscopic approach. Although multiple studies have demonstrated an adequate radiographic decompression, none has quantified the volume of bone removal after endoscopic decompression. The objective of this study was to quantify the 3D volume of bone removed from the lamina and facet joints during endoscopic decompression for lumbar central and lateral recess stenosis.
\end{abstract}

METHODS This retrospective study included adults with lumbar spinal stenosis who underwent endoscopic decompression of a single level or 2 noncontiguous lumbar levels. Central stenosis on MRI was graded preoperatively and postoperatively using the Schizas scale. A computer program was developed in MATLAB to semiautomatically perform a $3 \mathrm{D}$ volumetric analysis of preoperative and postoperative lumbar CT scans. The volumetric percentage of bone removed from the lamina and facet joints ipsilateral and contralateral to the side of approach was quantified.

RESULTS Nineteen patients with 21 treated lumbar levels were included in the study. Preoperatively, the number of levels with Schizas stenosis grades B, C, and D were 5, 12, and 4, respectively. Stenosis grades improved postoperatively to grades A, B, C, and D for 17, 3, 1, and 0 levels, respectively. All levels improved by at least 1 stenosis grade. The volumetric percentage of laminar bone removed was $15.5 \%(95 \% \mathrm{Cl} 11.2 \%-19.8 \%, \mathrm{p}<0.001)$ from the ipsilateral lamina and $8.8 \%(95 \% \mathrm{Cl} 5.7 \%-11.8 \%, \mathrm{p}<0.001)$ from the contralateral lamina. The percentage of facet joint resection was $5.3 \%(95 \% \mathrm{Cl} 4.2 \%-6.4 \%, \mathrm{p}<0.001)$ and $4.3 \%(95 \% \mathrm{Cl} 2.2 \%-6.4 \%, p<0.001)$ for the ipsilateral and contralateral facet joints, respectively. Average pain scores, as measured by the visual analog scale, improved from 7.9 preoperatively to 2.2 by $3-10$ months postoperatively $(p<0.001)$.

CONCLUSIONS Endoscopic lumbar decompression achieves improvement in the radiographic grade of lumbar central stenosis with minimal bone removal from the lamina and facet joints. Future prospective studies are needed to validate the findings of this study with more comprehensive clinical outcomes.

https://thejns.org/doi/abs/10.3171/2019.2.FOCUS18617

KEYWORDS lumbar stenosis; lumbar laminectomy; volumetric analysis; endoscopic interlaminar approach; facet preservation; ligamentum flavum

$\mathrm{L}$ UMBAR spinal stenosis can be classified as developmental, degenerative, or a combination of both. ${ }^{4}$ Degenerative lumbar stenosis is the most common type of lumbar stenosis and can encompass a combination of central, lateral recess, and/or neuroforaminal stenosis. ${ }^{11}$ Stenosis can be caused by a combination of disc/ osteophyte complex, ligamentum flavum hypertrophy, facet hypertrophy, and spondylolisthesis, which may be superimposed on a congenitally narrow canal. ${ }^{11}$ After exhaustion of conservative measures, the surgical treatment of choice when there is no concern for instability is lumbar decompression, which can be performed via open, tubular microscopic, or endoscopic means. Due to the use of angled scopes and the ability to change approach angles,

ABBREVIATIONS VAS $=$ visual analog scale.

SUBMITTED November 12, 2018. ACCEPTED February 26, 2019.

INCLUDE WHEN CITING DOI: 10.3171/2019.2.FOCUS18617.

${ }^{*}$ S.S.K. and H.S.K. share first authorship of this work. 
endoscopic decompression theoretically allows for minimizing the amount of bone removal needed to decompress the neural structures. ${ }^{6}$ In the case of central and lateral recess stenosis, decompression involves the lamina and the superior and inferior articulating processes of the bilateral facet joints at the level of decompression. Minimizing the amount of bone that is removed from the facet joints has the theoretical benefit of decreasing the incidence of postoperative instability. ${ }^{9}$

Three groups have demonstrated adequate decompression of the thecal sac after endoscopic laminectomy for central stenosis..$^{5,20,22}$ However, no studies have quantified the volume of lamina that is removed or the volume of facet destruction that occurs after endoscopic laminectomy for concomitant central and lateral recess stenosis. In this study, we sought to analyze the radiographic outcomes of percutaneous endoscopic lumbar spinal stenosis decompression and to quantify the volume of laminar and facet joint bone required to achieve neural decompression. We hypothesized that adequate radiographic neural decompression could be achieved endoscopically with minimal removal of bone from the lamina and facet joints.

\section{Methods}

\section{Patient Selection}

The Nanoori Gangnam Hospital institutional review board approved this retrospective study. Inclusion criteria were all adult patients who underwent decompression of a single lumbar level or multiple noncontiguous levels (for example, L2-3 and L4-5 in the same operation) for central and lateral recess stenosis. Patients with isolated lateral recess stenosis without central stenosis were excluded from the study. Patients with disc/osteophyte complexes were included only if discectomy was not performed at the time of central decompression. Patients with neuroforaminal stenosis that was specifically decompressed were excluded, as these patients require increased partial facet removal to obtain a far-lateral decompression, which falls outside the scope of our research question. Patients with Schizas grade B stenosis or worse on preoperative MRI were included in the study. ${ }^{18}$ All included patients underwent both preoperative and postoperative MRI and thin-cut CT scanning. Postoperative scans were obtained within 2 days of the operation. Any patients without both pre- and postoperative CT and MRI were excluded.

\section{Operative Technique}

We performed a bilateral lumbar decompression through a unilateral approach as described previously. ${ }^{9}$ In our practice, we refer to this procedure as "percutaneous endoscopic spinal stenosis lumbar decompression." Briefly, the patient was positioned prone on a Wilson frame. Most patients were placed under a combination of conscious sedation and epidural anesthesia. In rare cases, general anesthesia was utilized. A $1.5-\mathrm{cm}$ incision was centered on the lateral border of the interlaminar space at the level of planned decompression. An obturator was docked on this point, which corresponded to the superior aspect of the lateral lower lamina. An endoscopic working channel was placed, and the obturator was removed. An
iLESSYS Delta Endoscopic System (Joimax GmbH) was then inserted in the working channel.

The decompression was then performed in steps. First, bony structures were drilled on the ipsilateral side to expose the attachment of the ligamentum flavum cranially and caudally. Then, the ipsilateral outer layer of the ligamentum flavum was removed. Third, the contralateral bony structures were removed to expose the attachment of the ligamentum flavum on the cranial and caudal laminae. Fourth, the contralateral outer ligamentum flavum was removed. Fifth, the midline of the inner layer of the ligamentum flavum was exposed. Sixth, the contralateral inner layer of ligamentum flavum was removed to decompress the contralateral central canal and lateral recess. Lastly, the ipsilateral inner layer of ligamentum flavum was resected to complete the lateral recess decompression on the ipsilateral side. A small round drain was placed in some cases, depending on the level of hemostasis. The wound was closed with absorbable suture at the dermal layer and skin glue at the skin.

\section{Evaluation of Degree of Stenosis on MRI}

Due to its recognition in the published literature and demonstrated interobserver reliability, the Schizas scale was used in this study as a measure of spinal stenosis on MRI. ${ }^{1,15,16,18}$ In the Schizas system, grade A stenosis is the mildest, with abundant CSF inside the dural sac. There are 4 subgroups within grade $\mathrm{A}$. In grade $\mathrm{B}$ stenosis, the rootlets occupy the entire dural sac but can still be individualized. In grade $\mathrm{C}$, no rootlets can be recognized but epidural fat can be visualized dorsally. Lastly, in grade D the thecal sac is obliterated and no epidural fat, CSF, or individual rootlets are visible. ${ }^{18}$

Two neurosurgeons who were not involved with the operations independently evaluated preoperative and postoperative MR images obtained in patients who fit the inclusion criteria. If there was any disagreement, a third surgeon acted as the tiebreaker. The degree of stenosis was evaluated at the level of the middisc, the cranial portion of the disc, and the caudal portion of the disc. The Schizas grade at the level with the worst degree of stenosis was utilized for this study on both preoperative and postoperative MR images. The kappa statistic for interrater reliability was calculated to determine the agreement between the 2 observers (VassarStats). A Fisher exact probability test was then performed to determine if there was a difference between the preoperative and postoperative numbers of patients in each stenosis grade category (VassarStats).

\section{CT Volumetric Analysis to Quantify Volume of Bone Resected From Lamina and Facet Joints}

Volumetric analysis of lumbar CT scans was performed using a custom computer program developed in MATLAB (Image Processing Toolbox, release 2018b; MathWorks, Inc.). The program was run by a neurosurgeon who was not involved in the surgeries. First, the postoperative axial lumbar CT was imported into the program in DICOM format. CT resolution was $0.35 \times 0.35 \mathrm{~mm}$ per pixel within each axial slice, with 2-mm spacing between 

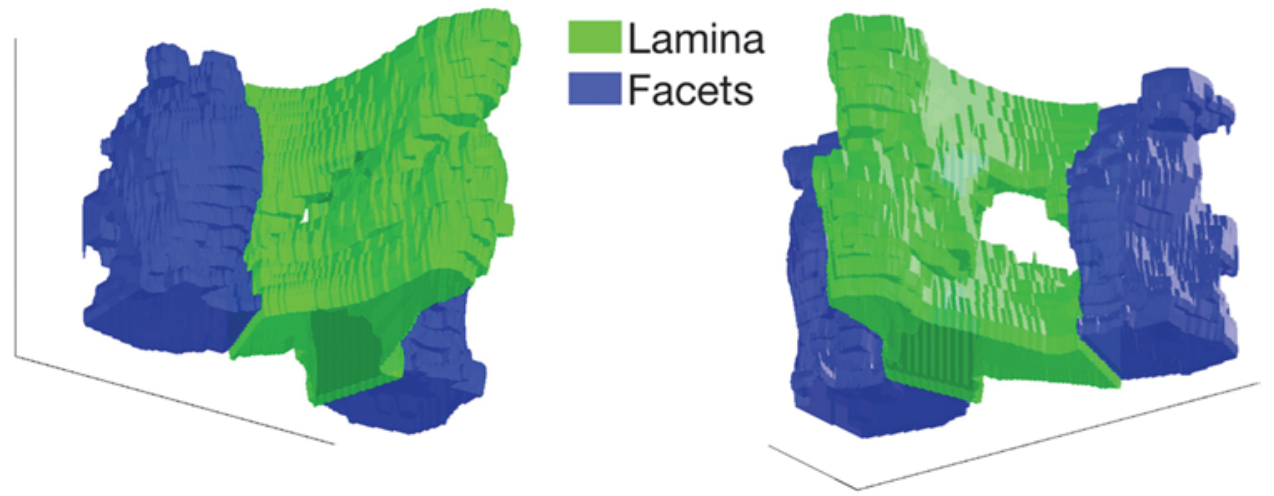

FIG. 1. Three-dimensional renderings of a postoperative CT scan at $L 4-5$, displaying the program's division of lamina and facets. Left: Contralateral to side of operative approach. Right: Ipsilateral to side of operative approach.

each slice. The axial CT images from the pedicle below to the pedicle above the level of decompression were included. Bone within these levels was isolated automatically with k-means clustering-based segmentation. ${ }^{3}$ The anatomical division of the segmented bone volume into isolated lamina and facet joints was performed with manually drawn regions of interest. Vertebral bodies, pedicles, and transverse processes were excluded. In our study, laminar volume included the spinous process. Overall, this algorithm yielded the number of bony voxels contained within the lamina and facet joints (Fig. 1).

The bone volume, in milliliters, was then calculated by multiplying number of voxels $\times$ pixel spacing in the leftright direction $\times$ pixel spacing in the anterior-posterior direction $\times$ spacing between $\mathrm{CT}$ slices (cranial-caudal direction). The relevant spacing-per-pixel quantities required for this calculation were obtained from the CT DICOM metadata.

The lamina and facet joint volumes were then calculated from the preoperative lumbar CT scan in a method similar to that described above. To ensure that the exact same anatomical divisions were used, the preoperative CT scan was automatically registered to the postoperative CT scan prior to volume calculation. A regular step gradient descent optimizer was used to calculate an affine transformation matrix from preoperative $\mathrm{CT}$ space to postoperative CT space. This operation ensured that the exact same anatomical divisions were used for the preoperative and postoperative CT scans.

The volumetric percentage of bone removal was calculated as $100 \times$ (preoperative bone volume - postoperative bone volume)/(preoperative bone volume).

Hypothesis testing for comparing preoperative and postoperative volumes was performed using the paired 2-tailed Student t-test.

\section{Clinical Outcomes}

Preoperative and postoperative visual analog scale (VAS) scores for pain were collected prospectively for each patient in the clinical setting., ${ }^{71}$ The postoperative pain score was recorded at the most recent postoperative visit, which ranged from 3 months to 10 months postoperatively (mean 5.9 months, standard deviation 1.9 months). Hypothesis testing to compare preoperative and postoper- ative VAS scores was performed with the paired 2-tailed Student t-test.

\section{Results}

Twenty-one patients with central canal and lateral recess stenosis met the inclusion criteria. Their endoscopic decompression surgeries were performed between February 2018 and September 2018, as our protocol to obtain thincut CT scans preoperatively and postoperatively started in February 2018. One of the 21 patients had a postoperative MRI that demonstrated a large, compressive, asymptomatic seroma. This patient was not included in the analysis due to the inability to assess ligamentous decompression. Another patient was excluded due to a poor-quality preoperative CT scan that was unsuitable for 3D model generation. The final cohort of 19 patients included 2 who underwent decompressive surgery at 2 noncontiguous levels. As such, 21 levels of decompression were analyzed: 1 at L1-2, 1 at $\mathrm{L} 2-3,5$ at $\mathrm{L} 3-4,13$ at $\mathrm{L} 4-5$, and 1 at $\mathrm{L} 5-\mathrm{S} 1$.

\section{Evaluation of Degree of Stenosis on MRI}

Utilizing the Schizas scale, there was excellent interrater reliability as demonstrated by a kappa statistic value of 0.93 (95\% CI 0.85-1). Preoperatively, there were 5 levels with Schizas grade B stenosis, 12 with grade C, and 4 with grade D. Postoperatively, there were 17 levels with grade A stenosis, 3 with grade B, and 1 with grade C. There were no levels with grade D stenosis postoperatively (Fig. 2). A comparison of pre- and postoperative MR images in a patient treated for central stenosis at L2-3 and L4-5 is provided in Fig. 3. There was a significant difference between the distributions of stenosis grades preoperatively and postoperatively $(\mathrm{p}<0.001)$. All patients and levels improved by at least 1 grade postoperatively. The 1 patient with a grade $\mathrm{C}$ stenosis postoperatively improved from grade $\mathrm{D}$ preoperatively, and his residual stenosis was partially due to an asymptomatic postoperative fluid collection.

\section{Volume of Bone Resected From Lamina and Facet Joints}

The results of the volumetric analysis are displayed in Fig. 4. The results were separated by side, ipsilateral and contralateral to the side of the operative approach. 


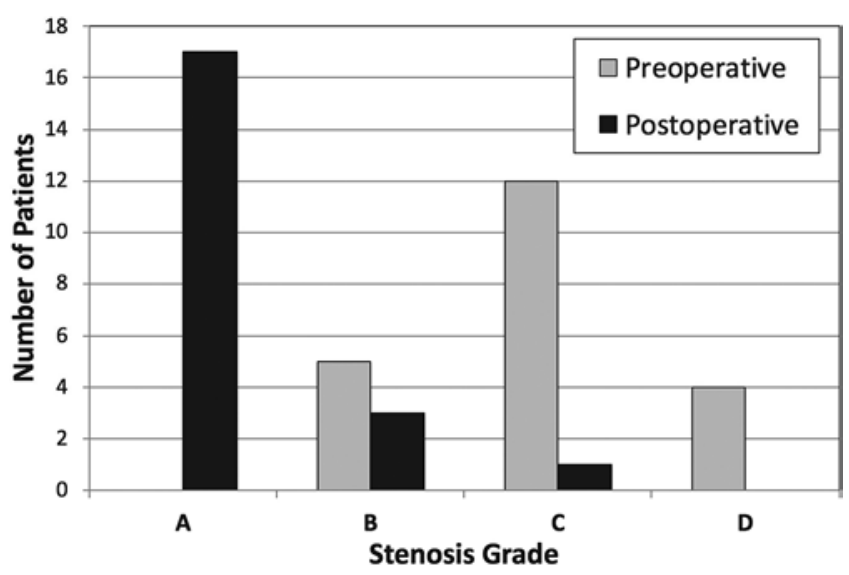

FIG. 2. Bar graph showing the number of patients with each Schizas lumbar stenosis grade preoperatively and postoperatively. All patients improved by at least 1 grade postoperatively, and most improved to Schizas grade A.

The volumetric percentage of laminar bone removed was $15.5 \%$ (95\% CI 11.2\%-19.8\%) from the ipsilateral lamina and $8.8 \%(95 \%$ CI $5.7 \%-11.8 \%)$ from the contralateral lamina. The percentage of facet joint resection was $5.3 \%$ (95\% CI 4.2\%-6.4\%) and 4.3\% (95\% CI 2.2\%-6.4\%) for the ipsilateral and contralateral facet joints, respectively. All volumetric comparisons were statistically significant $(\mathrm{p}<0.001)$.

\section{Clinical Outcomes}

Preoperatively, there were 3 patients with a VAS pain score of 10,2 patients with a score of 9,6 patients with a score of 8,7 patients with a score of 7 , and 1 patient with a score of 6 . All patients improved at least 4 levels. Postoperatively, there were 7 patients with a VAS score of 3 , 9 patients with a score of 2 , and 3 patients with a score of 1. The mean VAS score was 7.9 (95\% CI 7.4-8.5) preoperatively, which improved to 2.2 (95\% CI 1.9-2.5) postoperatively. This improvement was statistically significant ( $\mathrm{p}$ $<0.001)$.

\section{Discussion}

In this study, we showed that endoscopic lumbar decompression is efficacious in treating central and lateral recess stenosis, as measured by radiographic decompression on MRI and improvement in pain scores at followup. We also demonstrate via CT volumetric analysis that only a minimal amount of bone is removed from lamina and facet joints to achieve this decompression. With no control group undergoing tubular or open decompression, the goal of this project was not to state the benefit of endoscopic decompression over more conventional means, but to demonstrate that endoscopic decompressions can achieve their radiological objectives of adequate neural decompression with minimal laminar bone removal and negligible facet violation.

Ligamentum flavum hypertrophy was the primary pathology, which contributed to the central and lateral recess stenosis in the patients included in our study. This fact was central to our hypothesis that endoscopic decompression could achieve adequate decompression with minimal bone removal. A significantly larger volume of bone would need to be removed in cases in which bony compression is the primary pathology.

With regard to extent of decompression, our results confirm the results seen in 3 works in the literature showing adequate canal decompression after endoscopic lumbar procedures..$^{5,20,22}$ Dewanngan et al. ${ }^{5}$ demonstrated that endoscopic decompression was effective in improving anteroposterior diameter, interfacet distance, canal surface area, lateral recess height, and lateral recess angle measured on MRI. Zhou et al. ${ }^{22}$ noted improvement in the mean canal area and sagittal diameter of the lateral recess on CT scans obtained at 6 months and 2 years after surgery. Wada et al. ${ }^{20}$ noted improvement in the area of the dural tube at the level of compression to $408 \%$ after endoscopic decompression. Due to its excellent interobserver reliability and usage in the published literature, we utilized the Schizas qualitative measure, ${ }^{11,17,18}$ rather than quantitative measures such as dural sac cross-sectional area or stenosis ratio, to measure central stenosis on MRI.

This study is, to our knowledge, the first in the literature that systematically quantifies the volume of bone removal from the lamina and facet joints during endoscopic lumbar decompression. Utilizing CT volumetric analysis, we quantified the small amount of laminar bone that is removed to achieve adequate neural decompression. The cranial and caudal points for our model closely approximated the amount of bony decompression that many surgeons perform during an open laminectomy. Although we did not quantify the volume of bone removal during open laminectomy in this study, we hypothesize that endoscopic decompression requires significantly less bone removal than conventional laminectomy approaches. Endoscopy requires a minimal amount of bone removal due to the benefit of an expanded field of vision with an angled scope when compared with tubular and open procedures. $^{12}$

Facet-preserving decompression has the benefit of offering a less morbid alternative for patients with primarily leg symptoms and stenosis associated with spondylolisthesis or scoliosis..$^{8,12,14}$ Our study confirms that endoscopic decompression causes very little destruction to both the ipsilateral and contralateral facets. We do not have a comparison group to draw any conclusions about the benefit of this procedure over an open or tubular laminectomy in facet preservation. However, we do confirm the facetpreserving nature of endoscopic decompression via a novel method of measuring volume of facet joint. Typically, facet joints are measured in distances or areas, likely due to the difficulty of calculating volumes in commercially available software.,19 In our opinion, these methods of measuring facets do not accurately portray the amount of facet destruction sustained during surgery, as the facet is a 3D structure; measuring an area in a single plane can miss important information present in other planes. Therefore, we developed a custom computer program for volumetric analysis, capable of quantifying laminar and facet volume separately.

This retrospective study has multiple limitations. First, 
Khalsa et al.

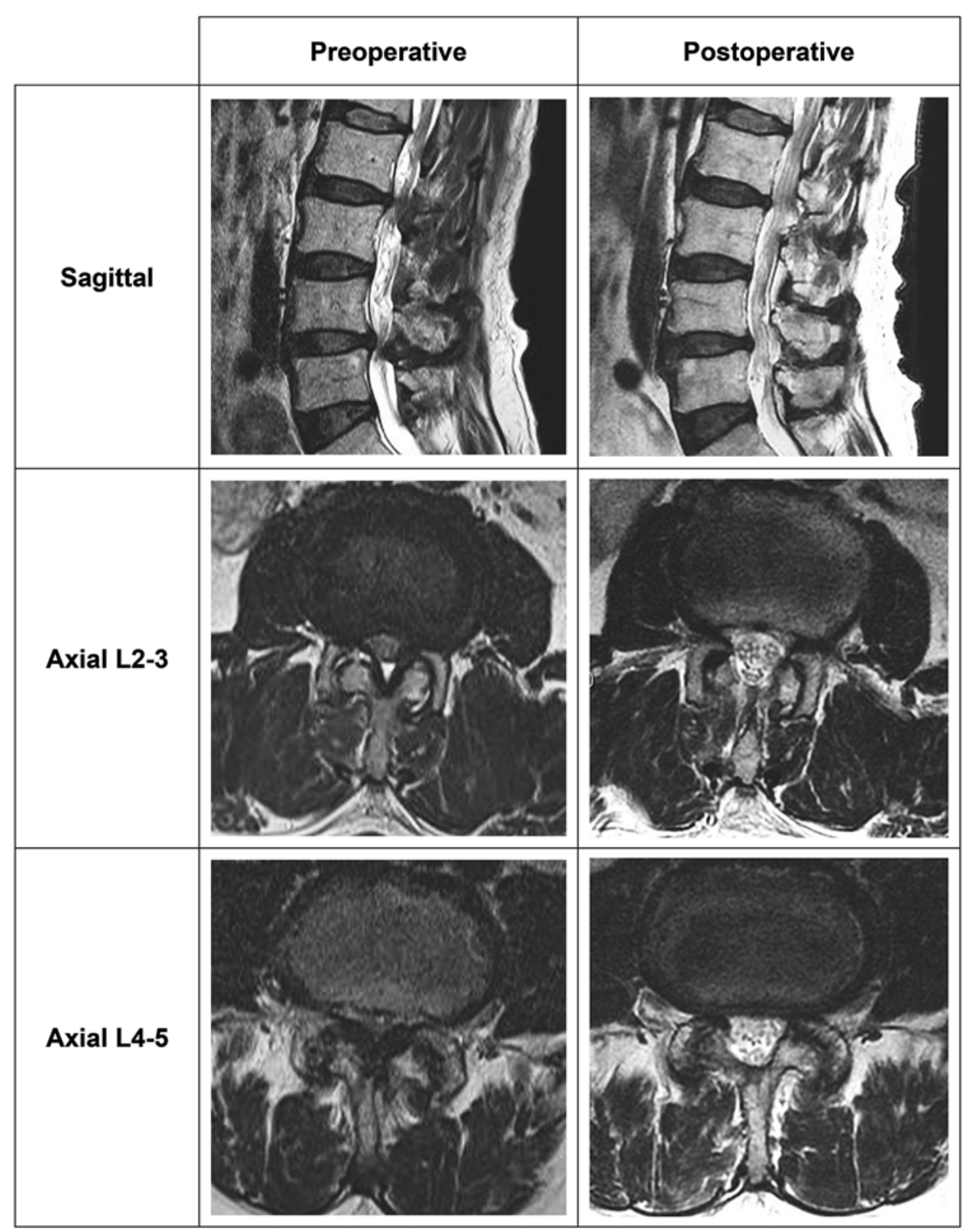

FIG. 3. A comparison of preoperative and postoperative MR images after endoscopic decompression for central stenosis at $L 2-3$ and L4-5. At L2-3, Schizas grade C improved to grade A. At L4-5, Schizas grade D improved to grade A.

clinical outcomes were limited to the VAS for pain. Our group plans to include more comprehensive clinical outcomes, such as the Oswestry Disability Index and SF-36, on a prospective basis in future studies. Even so, there is existing evidence in the literature demonstrating the clinical efficacy of endoscopic procedures for lumbar decompression. Endoscopic laminectomy has been shown to have equal long-term outcomes when compared with open surgery with the potential for decreased postoperative pain, complications, and hospital length of stay.10,12,13

Second, the study lacks a comparison group that underwent lumbar laminectomy via open or tubular means. However, the objective of this study was not to demonstrate the superiority of endoscopy over open or tubu- lar approaches, or vice versa. Lastly, the sample size is relatively small, due to the recent change in our clinical practice of obtaining thin-cut CT scans preoperatively and postoperatively. Even so, the results presented were all statistically significant.

\section{Conclusions}

Endoscopic lumbar decompression achieves improvement in the radiographic grade of lumbar central stenosis and improvement in pain scores with minimal bone removal from the lamina and facet joints. Performing an endoscopic lumbar decompression in patients with central and lateral stenosis allows for adequate neural decompres- 


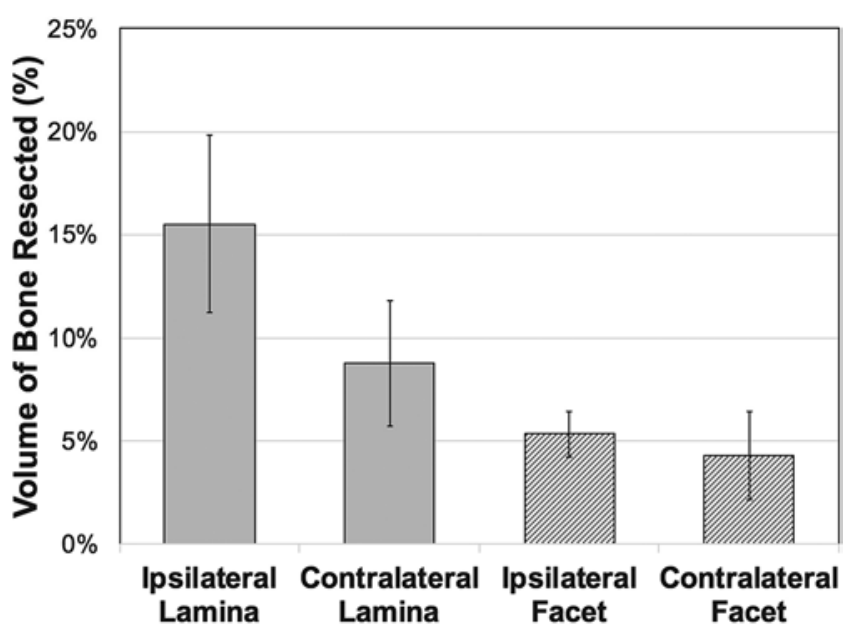

FIG. 4. Results of CT volumetric analysis illustrating the volumetric percentage of bone resected during endoscopic decompression. Minimal bone was resected from the lamina and facet joints. Results are separated ipsilateral and contralateral to the side of operative approach.

sion as measured by the Schizas scale. This decompression is achieved with minimal volume of laminar bone removal, and significant facet joint preservation, likely due to the superb illumination, clear visualization, and use of angled scopes. Future prospective studies are needed to validate the findings of this work with more comprehensive clinical outcomes.

\section{Acknowledgments}

We thank JaeEun Park for her clinical support and assistance with submission of the IRB application.

\section{References}

1. Alsaleh K, Ho D, Rosas-Arellano MP, Stewart TC, Gurr KR, Bailey CS: Radiographic assessment of degenerative lumbar spinal stenosis: is MRI superior to CT? Eur Spine J 26:362367,2017

2. An SJ, Seo MS, Choi SI, Lim TH, Shin SJ, Kang KN, et al: Facet joint hypertrophy is a misnomer: A retrospective study. Medicine (Baltimore) 97:e11090, 2018

3. Arthur D, Vassilvitskii S: k-means++: The advantages of careful seeding, in SODA 07: Proceedings of the Eighteenth Annual ACM-SIAM Symposium on Discrete Algorithms. New Orleans, 2007 (http://ilpubs.stanford. edu:8090/778/1/2006-13.pdf) [Accessed March 13, 2019]

4. Cheung JPY, Ng KKM, Cheung PWH, Samartzis D, Cheung KMC: Radiographic indices for lumbar developmental spinal stenosis. Scoliosis Spinal Disord 12:3, 2017

5. Dewanngan NK, Yadav YR, Parihar VS, Ratre S, Kher Y, Bhatele P: Extent of decompression of lumbar spinal canal after endoscopic surgery. J Neurol Surg A Cent Eur Neurosurg 78:541-547, 2017

6. Enyo Y, Yamada H, Kim JH, Yoshida M, Hutton WC: Microendoscopic lateral decompression for lumbar foraminal stenosis: a biomechanical study. J Spinal Disord Tech 27:257-262, 2014

7. Haro H, Maekawa S, Hamada Y: Prospective analysis of clinical evaluation and self-assessment by patients after decompression surgery for degenerative lumbar canal stenosis. Spine J 8:380-384, 2008

8. Kelleher MO, Timlin M, Persaud O, Rampersaud YR: Suc- cess and failure of minimally invasive decompression for focal lumbar spinal stenosis in patients with and without deformity. Spine (Phila Pa 1976) 35:E981-E987, 2010

9. Kim HS, Paudel B, Jang JS, Oh SH, Lee S, Park JE, et al: Percutaneous full endoscopic bilateral lumbar decompression of spinal stenosis through uniportal-contralateral approach: techniques and preliminary results. World Neurosurg 103:201-209, 2017

10. Kim JH, Kim HS, Kapoor A, Adsul N, Kim KJ, Choi SH, et al: Feasibility of full endoscopic spine surgery in patients over the age of 70 years with degenerative lumbar spine disease. Neurospine 15:131-137, 2018

11. Kitab S, Lee BS, Benzel EC: Redefining lumbar spinal stenosis as a developmental syndrome: an MRI-based multivariate analysis of findings in 709 patients throughout the 16- to 82year age spectrum. J Neurosurg Spine 29:654-660, 2018

12. Komp M, Hahn P, Oezdemir S, Giannakopoulos A, Heikenfeld R, Kasch R, et al: Bilateral spinal decompression of lumbar central stenosis with the full-endoscopic interlaminar versus microsurgical laminotomy technique: a prospective, randomized, controlled study. Pain Physician 18:61-70, 2015

13. Lee CH, Choi M, Ryu DS, Choi I, Kim CH, Kim HS, et al: Efficacy and safety of full-endoscopic decompression via interlaminar approach for central or lateral recess spinal stenosis of the lumbar spine: A meta-analysis. Spine (Phila Pa 1976) 43:1756-1764, 2018

14. Lee S, Srikantha U: Spinous Process splitting laminectomy: clinical outcome and radiological analysis of extent of decompression. Int J Spine Surg 9:20, 2015

15. Lønne G, Ødegård B, Johnsen LG, Solberg TK, Kvistad KA, Nygaard OP: MRI evaluation of lumbar spinal stenosis: is a rapid visual assessment as good as area measurement? Eur Spine J 23:1320-1324, 2014

16. Lurie JD, Tosteson AN, Tosteson TD, Carragee E, Carrino JA, Kaiser J, et al: Reliability of readings of magnetic resonance imaging features of lumbar spinal stenosis. Spine (Phila Pa 1976) 33:1605-1610, 2008

17. Mannion AF, Fekete TF, Pacifico D, O'Riordan D, Nauer S, von Büren M, et al: Dural sac cross-sectional area and morphological grade show significant associations with patientrated outcome of surgery for lumbar central spinal stenosis. Eur Spine J 26:2552-2564, 2017

18. Schizas C, Theumann N, Burn A, Tansey R, Wardlaw D, Smith FW, et al: Qualitative grading of severity of lumbar spinal stenosis based on the morphology of the dural sac on magnetic resonance images. Spine (Phila Pa 1976) 35:19191924,2010

19. Simon P, Espinoza Orías AA, Andersson GB, An HS, Inoue $\mathrm{N}$ : In vivo topographic analysis of lumbar facet joint space width distribution in healthy and symptomatic subjects. Spine (Phila Pa 1976) 37:1058-1064, 2012

20. Wada K, Sairyo K, Sakai T, Yasui N: Minimally invasive endoscopic bilateral decompression with a unilateral approach (endo-BiDUA) for elderly patients with lumbar spinal canal stenosis. Minim Invasive Neurosurg 53:65-68, 2010

21. Zanoli G, Strömqvist B, Jönsson B: Visual analog scales for interpretation of back and leg pain intensity in patients operated for degenerative lumbar spine disorders. Spine (Phila Pa 1976) 26:2375-2380, 2001

22. Zhou X, Zhang L, Zhang HL, He SS, Gu X, Gu GF, et al: Clinical outcome and postoperative CT measurements of microendoscopic decompression for lumbar spinal stenosis. Clin Spine Surg 30:243-250, 2017

\section{Disclosures}

Dr. Kim: consultant for Joimax. 


\section{Author Contributions}

Conception and design: all authors. Acquisition of data: Kashlan, Khalsa, Kim. Analysis and interpretation of data: all authors.

Drafting the article: Kashlan, Khalsa. Critically revising the

article: all authors. Reviewed submitted version of manuscript: all authors. Approved the final version of the manuscript on behalf of all authors: Kashlan. Administrative/technical/material support: Khalsa. Study supervision: Kashlan, Kim.

\section{Supplemental Information}

\section{Previous Presentations}

This work was presented as an oral presentation at Spine Summit 2019-35th Annual Meeting of the Section on Disorders of the Spine and Peripheral Nerves, Miami Beach, Florida, March 14-17, 2019, and in abstract form at the 2019 AANS Annual Scientific Meeting, San Diego, California, April 13-17, 2019.

\section{Correspondence}

Osama Nezar Kashlan: University of Michigan, Ann Arbor, MI. okashlan@med.umich.edu. 